\title{
High-Mobility Group AT-Hook I Served as a Prognosis Biomarker and Associated with Immune Infiltrate in Hepatocellular Carcinoma
}

\author{
Yong-Guang Wei \\ Cheng-Kun Yang \\ Zhong-Liu Wei \\ Xi-Wen Liao \\ Yong-Fei $\mathrm{He}$ \\ Xin Zhou \\ Hua-Sheng Huang \\ Chen-Lu Lan \\ Chuang-Ye Han \\ Tao Peng (D)
}

Department of Hepatobiliary Surgery, The First Affiliated Hospital of Guangxi Medical University, Nanning, 53002I, Guangxi Zhuang Autonomous Region, People's Republic of China
Correspondence: Tao Peng

Department of Hepatobiliary Surgery,

The First Affiliated Hospital of Guangxi

Medical University, Shuang Yong Road 6,

Nanning, Guangxi Zhuang Autonomous

Region, People's Republic of China

Tel +86-77l-5356528

Fax +86-77I-535003I

Email pengtaogmu@I63.com
Background: The protein high-mobility group AT-hook 1 (HMGA1) has been demonstrated that modulated cellular proliferation, invasion, and apoptosis with a poor prognosis in miscellaneous carcinomas. However, the mechanism of circumstantial carcinogenesis and association with the immune microenvironment of HMGA1 in hepatocellular carcinoma (HCC) had not been extensively explored.

Methods: The gene expression, clinicopathological correlation, and prognosis analysis were performed in the data obtained from TCGA. The results were further validated by ICGC and GEO database and external validation cohort from Guangxi. The HMGA1 protein expression was further examined in the HPA database. Biological function analyses were conducted by GSEA, STRING database, and Coexpedia online tool. Using TIMER and CIBERSORT method, the relationship between immune infiltrate and HMGA1 was investigated.

Results: In HCC, HMGA1 had much higher transcriptional and proteomic expression than in corresponding paraneoplastic tissue. Patients with high HMGA1 expression had a poor prognosis and unpromising clinicopathological features. High HMGA1 expression was closely related to the cell cycle, tumorigenesis, substance metabolism, and immune processes by regulating complex signaling pathways. Notably, HMGA1 may be associated with TP53 mutational carcinogenesis. Moreover, increased HMGAl expression may lead to an increase in immune infiltration and a decrease in tumor purity in HCC. CIBERSORT analysis elucidated that the amount of B cell naive, B cell memory, T cells gamma delta, macrophages M2, and mast cell resting decreased when HMGA1 expression was high, whereas T cells follicular helper, macrophages M0, and Dendritic cells resting increased.

Conclusion: In conclusions, HMGA1 is a potent prognostic biomarker and a sign of immune infiltration in HCC, which may be a potential immunotherapy target for HCC.

Keywords: hepatocellular carcinoma, HMGA1, prognostic signature, bioinformatics, immune filtration

\section{Introduction}

Hepatocellular carcinoma (HCC), which accounts for $75-85 \%$ of all cases, is the predominant type of liver cancer with a highly malignant nature due to its insidious onset, rapid progression, and intrahepatic and distant metastasis. ${ }^{1,2}$ Liver cancer is one of the most common malignancies and the second most frequent cause of cancer-associated mortality worldwide. More than $80 \%$ of HCC cases are estimated to occur in the countries with low-medium resources, particularly in Eastern Asia and sub-Saharan Africa. ${ }^{1}$ An investigation showed that liver cancer was one of the five leading causes 
of years of life lost (YLLs) in 2017 in China, suggesting it had brought an grave burden to society. ${ }^{3}$

Over the past few decades, there has been considerable progress in comprehending the epidemiology, risk factors, and molecular profiles of HCC. On the etiology, HCC arises from infection of chronic hepatitis (B and $\mathrm{C}$ ) virus and cirrhosis, excessive drinking, metabolic liver diseases, particularly nonalcoholic fatty liver disease (NAFLD), prolonged dietary toxins exposure such as aflatoxin and aristolochic acid, type 2 diabetes mellitus, obesity, and smoking., These ongoing risk factors mediate the liver cells damage, chronic inflammation, and further genomic changes. Indeed, HCC can actually be clinically cured with an early diagnosis with a satisfactory prognosis when potentially curative approaches are applicable. ${ }^{1,6}$ In recent years, the researchers have made headway on the operative treatment and medication of HCC. ${ }^{7-11}$ Immune checkpoint inhibitor (ICI) therapy has become an incrementally utilized treatment modality across the various stages of HCC. This provides a new therapeutic prospect for patients with $\mathrm{HCC}$ and underscores the crucial role of the tumor microenvironment (TME) in the development of HCC. ${ }^{12-15}$ Unfortunately, due to the complexity of immunotherapy and the genetic heterogeneity, this method took effect only for partial patients. ${ }^{16}$ Emerging evidence demonstrated that the change of tumor-induced immune infiltrate cells was a momentous mechanism that carcinomas develop to evade immune surveillance. Meanwhile, tumor immune infiltration carries a clinicopathological significance in predicting prognosis and therapeutic efficacy. ${ }^{17-19}$

HMGA1 is a non-histone nuclear protein as well as a chromatin architectural protein. In contrast to the high expression levels in various carcinomas, HMGA1 expression levels in normal tissue were low or undetectable. Several studies demonstrated that a high level of HMGA1 expression correlated with adverse clinical outcomes and more advanced disease in cancers. ${ }^{20-23}$ Previous researches have shown that HMGA1 connected with hepatitis B virusmediated changes and liver fibrosis. ${ }^{24,25}$ Further, it acted as an oncogenic driver of progression and migration with a poor prognosis in HCC. ${ }^{26,27}$ Higher HMGA1 mRNA and protein levels were discovered in HCCs with intrahepatic metastases compared to those without intrahepatic metastases, indicating the aggressive nature of HMGAl in HCC development. ${ }^{28,29}$ The reviews concluded that the ability to modulate gene expression and chromatin remodeling led to complex biological activities of HMGA1, resulting in the transformation of different molecular pathways. ${ }^{23,30-32}$
Despite this, the exact mechanisms of HMGA1 on hepatocarcinogenesis and its immune relevance remain to be further elucidated to lay the foundation for the next analysis at a deeper level. Therefore, this study comprehensively evaluated the prognosis significance, latent biological functions, and the association with immune infiltrating cells of HMGA1 in $\mathrm{HCC}$.

\section{Materials and Methods Hepatocellular Carcinoma (HCC) Datasets and Data Processing}

The Cancer Genome Atlas (TCGA) database portal (https:// portal.gdc.cancer.gov/cart; up to May 15, 2019) was employed to retained data of HCC samples on gene expression (Workflow Type: HT Seq-FPKM), clinical features (Data Type: Clinical Supplement), and genetic mutation. Samples lacking information on survival time, pathological grade, lymphatic, and distant metastasis were excluded. Processed RNASeq FPKM data of 374 pathologically verified HCC and 50 adjacent normal tissues were included. In addition, 232 HCC patients were included in The combined International Cancer Genomics Consortium (ICGC) cohort, and the Gene Expression Omnibus (GEO) cohort (GSE14520 datasets) included $212 \mathrm{HCC}$ patients. Their expression and the clinical information was respectively obtained from the ICGC (http:// dcc.icgc.org) and GEO database (https://www.ncbi.nlm.nih. gov/geo/). For the Kaplan-Meier survival analysis, the patients were divided into high- and low-HMGA1 expression groups according to the optimal cutoff value figured out by a userfriendly and web-based integrative tool (ESurv, https://www. giantonline.org/). ${ }^{33}$

\section{Differential mRNA Expression, Prognosis Value, and Clinicopathological Correlation Analyses}

The differentially expressed HMGA1 in HCC and normal tissues were detected using the Wilcoxon test method. To investigate the prognostic value of HMGA1, the KaplanMeier analysis was performed to compare overall survival (OS) time between high- and low-HMGA1 expression groups. Log rank test was used for statistical comparison. The log-rank $\mathrm{p}$-values and the hazard ratios were figured. Univariate Cox proportional hazards regression and multivariate Cox regression analyses were utilized to assess the relationship between HMGA1 and OS time. The receiver operating characteristic (ROC) curve was also implemented. Logistic regression analysis was utilized to figure out whether there are significant 
variances in HMGA1 expression under different clinicopathological statuses. The associations between HMGA1 expression and certain clinicopathological parameters were analyzed using the Student's $t$-test.

\section{Biological Function Analysis}

To gain insight into the biological processes associated with the HMGA1 regulatory network that could underlie HCC development, Gene Ontology (GO) and Kyoto Encyclopedia of Genes and Genomes (KEGG) analyses were performed based on normalized RNA-Seq data obtained from the TCGA dataset using Gene Set Enrichment Analysis (GSEA). The count of permutations was set to 1000 . Functional categories with a false discovery rate (FDR) < 0.050 and a nominal p-value $<0.050$ were regarded as statistically significant pathways. Furthermore, we explored the STRING (search tool for recurring instances of neighboring genes) website (https://string-db.org/) to construct a proteinprotein interaction (PPI) network among HMGAl and the neighboring 10 genes significantly associated with it. An interaction score of 0.4 was set as a cut-off value. ${ }^{34}$ The HMGA1 co-expression network was assessed utilizing Coexpedia online tools (http://www.coexpedia.org/) and was reconstructed by Cytoscape. ${ }^{35}$ To examine the possible mechanism by which HMGA1 acts on the p53 signaling pathway, Pearson correlation analyses were performed on HMGA1 and some TP53-related genes respectively.

\section{Immune Infiltrates Analysis}

TIMER (Tumor Immune Estimation Resource) is a comprehensive resource, based on statistical computation known as deconvolution, ${ }^{36}$ which is used to perform interactive analysis of the associations between the abundance of immune infiltrates and gene expression level (https://cis trome.shinyapps.io/timer/). ${ }^{37}$ Using TIMER, the abundance of six tumor-infiltrating immune cells (TIICs), including B cells, CD4 T cells, CD8 T cells, macrophages, neutrophils, and dendritic cells as well as infiltrate purity was approximated. Besides, CIBERSORT is an analytical tool that estimates the abundance of member cell types in a mixed cell population using gene expression data (http://cibersort.stan ford.edu/). ${ }^{38}$ Through the CIBERSORT (Cell type Identification through the Estimating Relative Subsets of RNA Transcripts) algorithm, we evaluated the immune response of 22 TIICs in HCC based on the TCGA dataset and then evaluated their abundance between the high and low HMGA1 expression groups. A p-value $<0.05$ makes known the statistical significance of deconvolution outcomes.

\section{Collection and Reverse}

Transcription-Quantitative PCR (RT-qPCR) of HCC Samples in the Guangxi Cohort

From 2017 to 2018, surgical biopsies were collected from 29 patients who underwent curative hepatic resections for HCC at The First Affiliated Hospital of Guangxi Medical University (Guangxi, China) without the preoperative therapies. OS information was retrieved through electronic medical records or telephone follow-up. All patients enrolled signed informed consent forms to donate their tissue samples for biomedical research, which was authorized by the ethics committee of The First Affiliated Hospital of Guangxi Medical University [Approval Number: 2021 (KY-E-032)]. In our previous study, the way of specimen preservation and the specific procedures of RNA extraction and reverse transcriptionquantitative PCR had been mentioned. ${ }^{39}$ The sequence of primers was as following: GAPDH, forward GTCAGCCGCATCTTCTTT, reverse CGCCCAATAC GACCAAAT. HMGA1, forward AAACC AAGGGGCAGACCCAA, reverse CTGTGTA GTGTGGTGGTGAGG. Using the $2-\Delta \Delta C t$ method, the expression levels of HMGA1 were estimated to conduct validation analysis.

\section{Validation of Differential Expression, Prognosis Value of HMGAI}

The differential expression in HCC vs para-carcinoma tissues and prognosis value of HMGA1 were further validated by investigating the data from ICGC, GSE14520, and Guangxi cohorts. Furthermore, the HPA database (https://www.protei natlas.org) was used to exam the protein expression of HMGA1 and to accurately assess protein localization.

\section{Statistical Analysis}

Unless otherwise noted, all analyses were performed using $\mathrm{R}$ software (version 3.6.3) and p-value $<0.05$ was considered to be significant.

\section{Result}

\section{Discrepant mRNA and Protein Expression of HMGAI}

The HMGA1 expression levels were significantly upregulated in HCC tissues in comparison with the non-tumor controls (Figure 1A, $\mathrm{P}<0.001$ ). Such result was further verified by mining Guangxi (Figure 1B, P $<0.001$ ), ICGC 

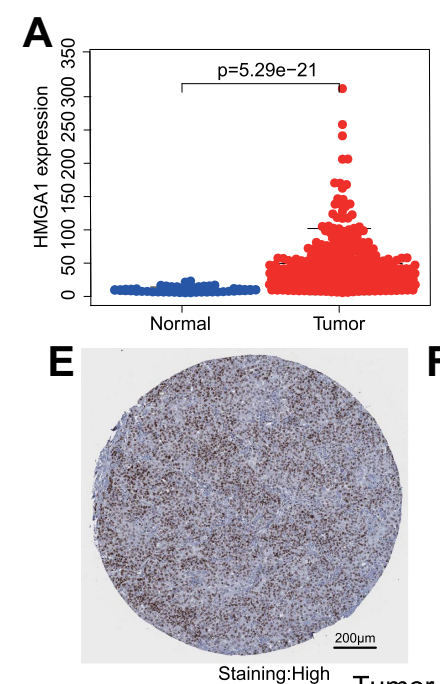

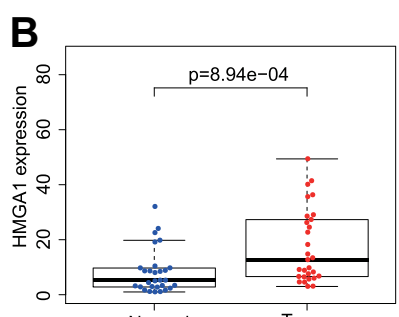

$\mathbf{F}$

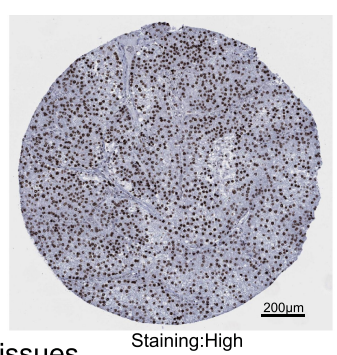

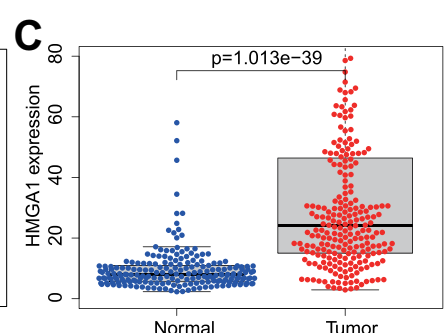

$\mathbf{G}$

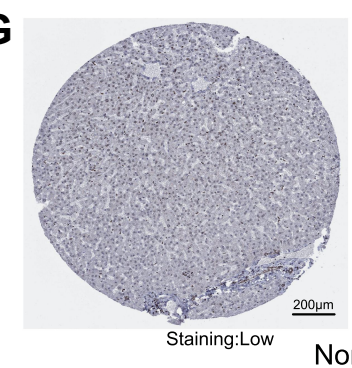

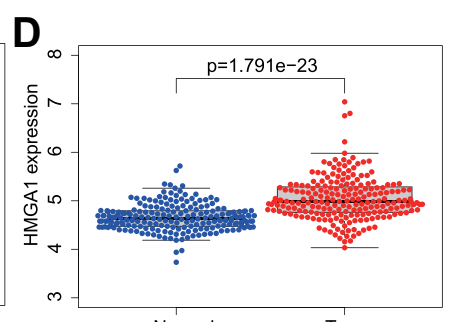

H

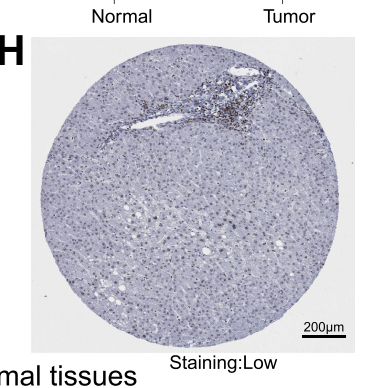

Figure I HMGAI mRNA expression levels in normal and HCC tissues, as obtained from the (A) TCGA, (B) ICGC, (C) GEO, and (D) Guangxi cohort. Representative immunohistochemical staining images of HMGAI protein in HCC specimens (E and $\mathbf{F}$ ) and in normal liver tissues $(\mathbf{G}$ and $\mathbf{H})$, which taken from the open HPA dataset. Abbreviations: HMGAI, high-mobility group AT-hook I; HCC, hepatocellular carcinoma; TCGA, The Cancer Genome Atlas; ICGC, International Cancer Genomics Consortium; GEO, Gene Expression Omnibus; HPA, The Human Protein Atlas.

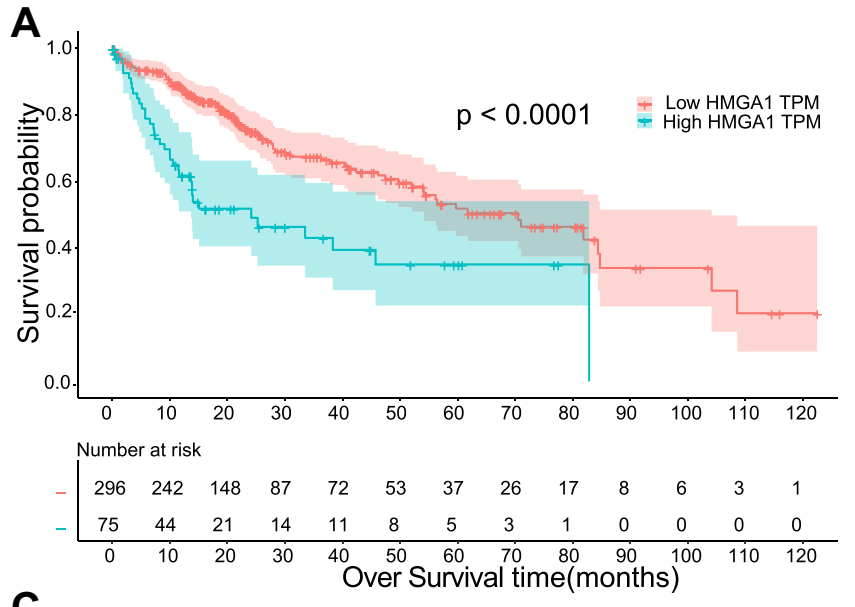

C

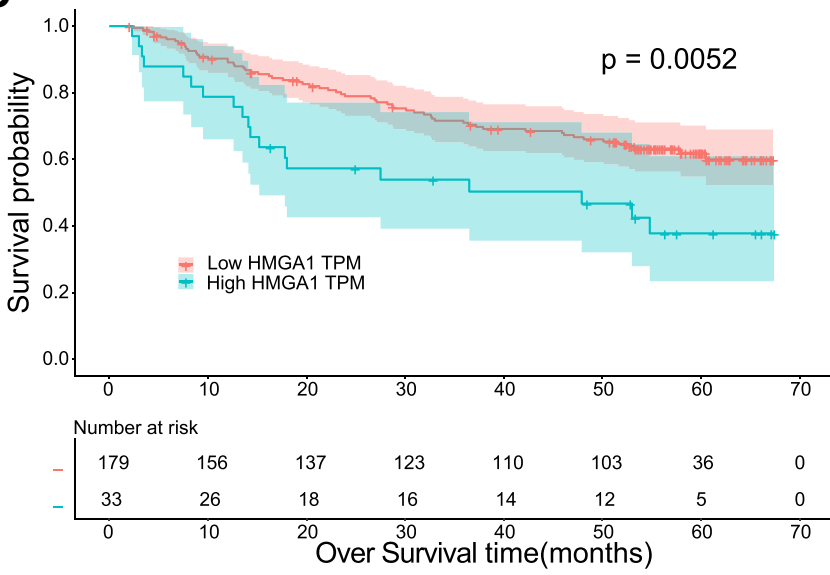

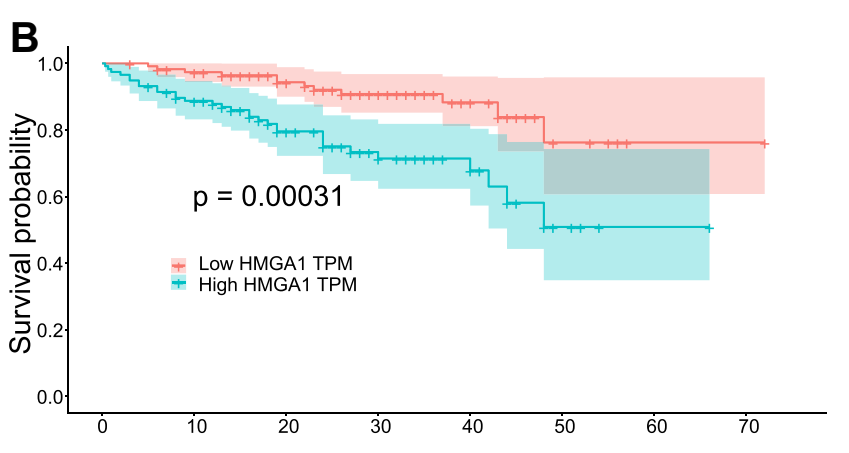

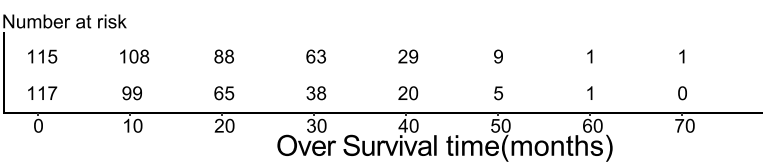

D

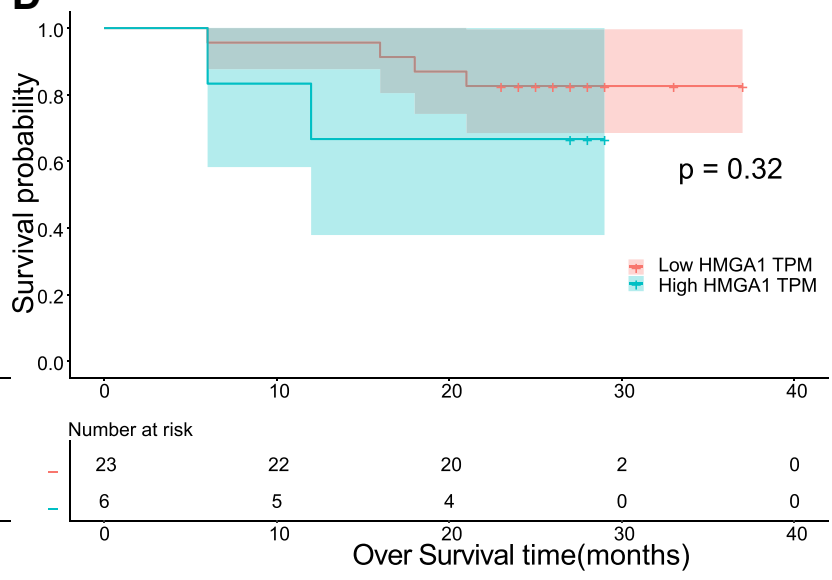

Figure 2 Kaplan-Meier analysis of HMGAI and OS time of HCC in the (A) TCGA, (B) ICGC, (C) GEO, and (D) Guangxi cohorts.

Abbreviations: HMGAI, high-mobility group AT-hook I; HCC, hepatocellular carcinoma; OS, overall survival; TCGA, The Cancer Genome Atlas; ICGC, International Cancer Genomics Consortium; GEO, Gene Expression Omnibus. 
(Figure 1C, P < 0.001), and GSE14520 (Figure 1D, P < 0.001) cohorts. Consistent with the mRNA expression analysis, the proteins encoded by HMGA1 were strongly positive in HCCs (Figure 1E and F), while were low-expression in normal liver tissues in the human Protein Profiles (Figure $1 \mathrm{G}$ and $\mathrm{H}$ ). Besides, HMGA1 protein was mainly localized to nuclei.

\section{Prognosis Value and Clinicopathological Correlation of HMGAI}

In the TCGA cohort, the augmented expression of HMGA1 corresponded with worse OS at the optimal cut-off point of 54.73 (Figure 2A, $\mathrm{P}<0.001$ ). Consistently, the enhanced mRNA expression of HMGA1 linked to the poor OS in the ICGC (Figure 2B, $\mathrm{P}<0.05$ ) and GSE14520 cohorts (Figure $2 \mathrm{C}, \mathrm{P}<0.05$ ). Nevertheless, survival analysis was not statistically significant in Guangxi cohort, which may be due an insufficient sample size and adequate follow-up time (Figure 2D). The connections between HMGA1 expression and clinical information in patients with HCC were analyzed (Table 1). As for cancer status, HMGA1 upregulation expressions were prone to be found in the HCCs with poorer clinicopathological features, including histological grade, $\mathrm{T}$ stage, and pathological stage (Figure $3 \mathrm{~A}-\mathrm{C}$, all $\mathrm{P}<0.001$ ).

Table I Association Between Clinicopathological Features of Patients and HMGAI Expression in the Cancer Genome Atlas

\begin{tabular}{|c|c|c|c|c|}
\hline $\begin{array}{l}\text { Clinicopathological } \\
\text { Parameters }\end{array}$ & Cases(n) & $\begin{array}{c}\text { HMGAI } \\
\text { Expression } \\
\text { Levels }\end{array}$ & $\mathbf{T}$ & P-value \\
\hline \multicolumn{5}{|l|}{ Age } \\
\hline$<60$ years & 125 & 43.01 & 1.342 & 0.181 \\
\hline$\geq 60$ years & 110 & 32.00 & & \\
\hline \multicolumn{5}{|l|}{ Gender } \\
\hline Male & 161 & 39.48 & -0.234 & 0.815 \\
\hline Female & 74 & 38.22 & & \\
\hline \multicolumn{5}{|l|}{ Stage } \\
\hline $1+I I$ & 163 & 35.65 & -2.081 & $0.039 *$ \\
\hline III+IV & 72 & 46.85 & & \\
\hline \multicolumn{5}{|l|}{ Grade } \\
\hline $\mathrm{G} 1+\mathrm{G} 2$ & 132 & 32.57 & -2.858 & $0.005^{*}$ \\
\hline $\mathrm{G} 3+\mathrm{G} 4$ & 103 & 47.43 & & \\
\hline \multicolumn{5}{|l|}{ T stage } \\
\hline $\mathrm{TI}+\mathrm{T} 2$ & 167 & 35.68 & -1.800 & 0.075 \\
\hline $\mathrm{T} 3+\mathrm{T} 4$ & 68 & 47.44 & & \\
\hline \multicolumn{5}{|l|}{$N$ stage } \\
\hline No & 231 & 38.58 & 0.252 & 0.801 \\
\hline $\mathrm{NI}$ & 4 & 19.81 & & \\
\hline \multicolumn{5}{|l|}{ M stage } \\
\hline Mo & 231 & 38.61 & 0.195 & 0.845 \\
\hline MI & 4 & 13.68 & & \\
\hline
\end{tabular}

Note: *Statistically significant.
Interestingly, HMGA1 expression showed a monotone increasing from histological grade I to IV, pathological stage I to III, and T1 stage to T3 stage. Nevertheless, HMGA1 expression in pathological stage IV or T4 stage is not the highest. This may be attributed to the cause that operation should not be considered as a major treatment measure for advanced-stage patients. Logistic regression analysis demonstrated that T stage (II and III vs I), stage (II and III vs I) and grade (II and III vs I) were significantly associated with increased HMGAl expression level in HCC (Table 2). Furthermore, a notable discovery indicated that HCCs with TP53 mutations had higher HMGA1 expression levels in the TCGA (Figure 3D, P < 0.001). Univariate analysis indicated that pathological stage, $\mathrm{T}$ and $\mathrm{M}$ stage were markedly correlated with OS (Table 3, P $<0.05$ ). Following that, multivariate analysis indicated that HMGA1 overexpression remained as an independent predictive marker of OS (Table 2, Figure 3E, P < 0.001). Meanwhile, HMGA1 expression showed a promising prognostic power as the ROC curve illustrated that the area under the curve (AUC) of HMGA1 expression for predicting OS was 0.622 (Figure 3F).

\section{Biological Function of HMGA I}

According to the results of GO terms enrichment analysis, upregulated HMGA1 was significantly concentrated in "RNA catabolic process, mRNA binding, cell cycle G2/ $M$ phase transition, and mRNA processing" and downregulated HMGA1 was enriched in "cellular amino acid catabolic process, alpha-amino acid catabolic process, organic acid catabolic process, protein activation cascade, and complement activation alternative pathway" (Figure 4A). KEGG pathway analysis elucidated that the upregulated HMGA1 was mainly involved in "DNA replication, pathway in cancer, cell cycle, MAPK signaling pathway, NOTCH signaling pathway, VEGF signaling pathway, p53 signaling pathway, and WNT signaling pathway", while "complement and coagulation cascades, tryptophan metabolism, PPAR signaling pathway, fatty acid metabolism, and primary bile acid biosynthesis" were enriched in downregulated HMGA1 (Figure 4B). Moreover, a PPI network constructed from the STRING database showed that TP53, LMNB1, RB1, RPS6KB1, EP400, HMGCR, INSIG1, HMGA2, CEBPB, and C6orf1 proteins were significantly related to HMGA1 protein (Figure 4C). The HMGA1 co-expression network was analyzed and created by Coexpedia, in which heterogeneous nuclear ribonucleoprotein U-like 1 (hnRPUL1) carried the greatest correlation with the highest edges' LLS 

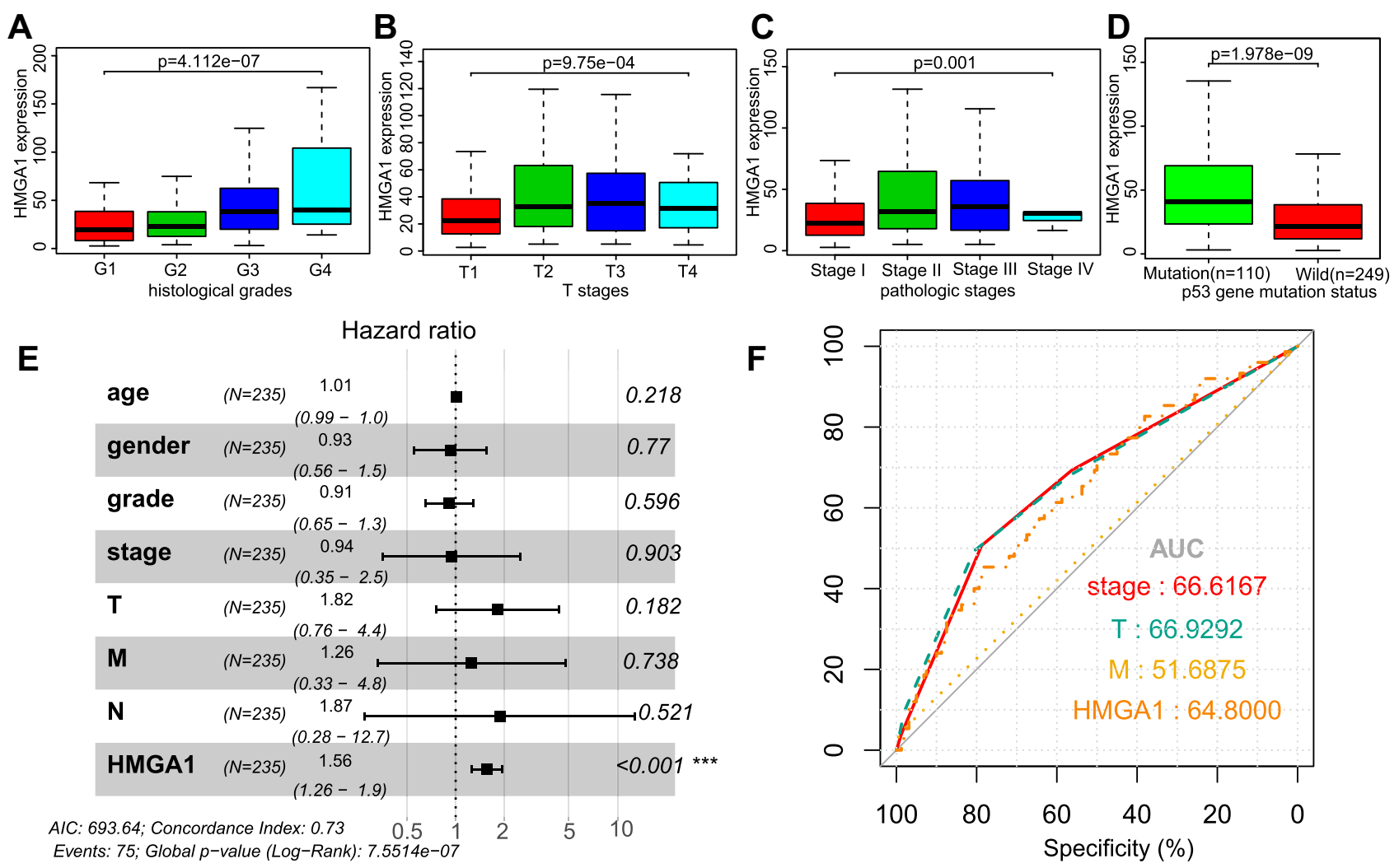

Figure 3 Boxplot showing expression of HMGAI correlate with different histological grades (A), T stages (B), pathologic stages (C), and p53 gene mutation status (D) in $\mathrm{HCC}$. The $t$-test was used to estimate the significance of the difference in gene expression levels between groups. The application value of HMGAI in HCC clinical parameters. (E) Forest plot illustrated the results of multivariate Cox regression analyses. ****Stands for $P<0.00 \mathrm{I}$. (F) ROC curves of pathologic stage, T stage, M stage, and HMGAI expression.

Abbreviations: HMGAI, high-mobility group AT-hook I; HCC, hepatocellular carcinoma; ROC, Receiver operating characteristic; T stage, Tumor stage; M stage, Metastasis stage; AUC, Area under Curve.

(log-likelihood score) of 9.276 (Figure 4D). Furthermore, Pearson correlation analyses uncovered that HMGA1 was significant positive correlation to TP53 $(\mathrm{R}=0.4)$, RB1 $(\mathrm{R}=0.17), \quad \mathrm{CCNB} 1 \quad(\mathrm{R}=0.54), \quad$ PAK1 $\quad(\mathrm{R}=0.42), \quad \mathrm{CDK} 1$

Table 2 Association Between HMGAI Expression and Clinicopathological Features (Logistic Regression)

\begin{tabular}{|l|l|l|l|}
\hline Clinical Characteristic & Total(N) & $\begin{array}{l}\text { (OR) in HMGAI } \\
\text { Expression }\end{array}$ & P-value \\
\hline Age & 370 & $0.822(0.545-1.237)$ & 0.348 \\
Stage (II vs I) & 258 & $2.103(I .247-3.58 I)$ & $0.006^{*}$ \\
Stage (III vs I) & 256 & $2.387(I .407-4.095)$ & $0.00 I^{*}$ \\
Stage (IV vs I) & 176 & $2.272(0.367-17.587)$ & 0.376 \\
Grade (II vs I) & 234 & $0.414(0.213-0.776)$ & $0.007^{*}$ \\
Grade (III vs I) & 206 & $0.359(0.179-0.698)$ & $0.003^{*}$ \\
Grade (IV vs I) & 67 & $0.320(0.084-1.140)$ & $0.08 I$ \\
Tumor status (II vs I) & 275 & $2.496(I .503-4.192)$ & $<0.00 I^{*}$ \\
Tumor status (III vs I) & 26 I & $2.323(I .363-4.006)$ & $0.002^{*}$ \\
Tumor status (IV vs I) & 194 & $1.808(0.578-5.824)$ & 0.304 \\
Lymph node (II vs I) & 256 & $1.000(0.118-8.439)$ & 1.000 \\
Distant metastasis (II vs I) & 300 & $3.045(0.384-61.996)$ & 0.338 \\
\hline
\end{tabular}

Note: *Statistically significant.

Abbreviation: OR, odds ratio.
$(\mathrm{R}=0.37), \quad \mathrm{CDK} 2 \quad(\mathrm{R}=0.34), \quad \mathrm{CDK} 4 \quad(\mathrm{R}=0.55), \quad \mathrm{E} 2 \mathrm{~F} 1$ $(\mathrm{R}=0.22)$, E2F2 $(\mathrm{R}=0.39)$, HNRNPUL1 $(\mathrm{R}=0.45)$, while was negatively correlated to GADD45A $(\mathrm{R}=-0.23)$ (Figure 5A-L, all $\mathrm{P}<0.001$ ).

\section{Immune Cells Infiltration and HMGAI}

In TIMER, increased HMGA1 expression resulted in a rise in immune infiltration level, including infiltrated B cells, CD4+, CD8+ T cells, neutrophils, macrophages, and dendritic cells (DCs) in HCC, as well as a reduction in infiltrating purity (Figure 6A, all $\mathrm{P}<0.001$ ). Notably, macrophages had the highest correlation coefficient among these immune infiltrate cells. Moreover, CIBERSORT analysis elucidated that the amount of B cell naive, B cell memory, T cells gamma delta, Macrophages M2, and mast cell resting decreased when HMGA1 expression was high, whereas $\mathrm{T}$ cells follicular helper, Macrophages M0, and Dendritic cells resting increased (Figure 6B, $\mathrm{P}<0.05$ ). The resulting heat map manifested that there was a weak to moderate correlation among diverse TIICs subpopulations (Figure 7). 
Table 3 Univariate Cox Proportional Hazards Regression and Multivariate Cox Regression Analyses of HMGAI and Clinical Features in $\mathrm{HCC}$

\begin{tabular}{|l|c|c|c|c|c|c|}
\hline \multirow{2}{*}{ Variable } & \multicolumn{3}{|c|}{ Univariate Analysis } & \multicolumn{3}{c|}{ Multivariate Analysis } \\
\cline { 2 - 7 } & HR & $95 \%$ Cl & P-value & HR & $95 \%$ CI & P-value \\
\hline Age & 1.005 & $0.987-1.023$ & 0.591 & 1.012 & $0.993-1.032$ & 0.218 \\
Gender & 0.780 & $0.487-1.249$ & 0.301 & 0.927 & $0.556-1.545$ & 0.770 \\
Grade & 1.017 & $0.746-1.387$ & 0.914 & 0.913 & $0.652-1.279$ & 0.596 \\
Stage & 1.865 & $1.456-2.388$ & $<0.00 I^{*}$ & 0.941 & $0.353-2.508$ & 0.903 \\
T stage & 1.804 & $1.434-2.270$ & $<0.00 I^{*}$ & 1.816 & $0.756-4.360$ & 0.182 \\
N stage & 2.022 & $0.494-8.276$ & 0.328 & 1.872 & $0.275-12.743$ & 0.521 \\
M stage & 3.850 & $1.207-12.28 I$ & $0.023 *$ & 1.256 & $0.331-4.758$ & 0.738 \\
HMGAI & 1.571 & $1.285-1.922$ & $<0.00 I^{*}$ & 1.558 & $1.257-1.922$ & $<0.00 I^{*}$ \\
\hline
\end{tabular}

Note: $* P<0.05$ was considered statistically significant.

Abbreviations: $\mathrm{HR}$, hazard ratio; $\mathrm{Cl}$, confidence interval.

\section{Discussion}

Several studies have elucidated that high mobility group A1 (HMGA1), an important member of the HMGA family, played a central role in the pathogenesis and progression of diverse malignant tumors. ${ }^{23,40}$ HMGA1 is overexpressed during embryogenesis but at relatively low levels in normal adult tissues, implying that HMGA1 may act as a critical molecular switch to form transcriptional networks that
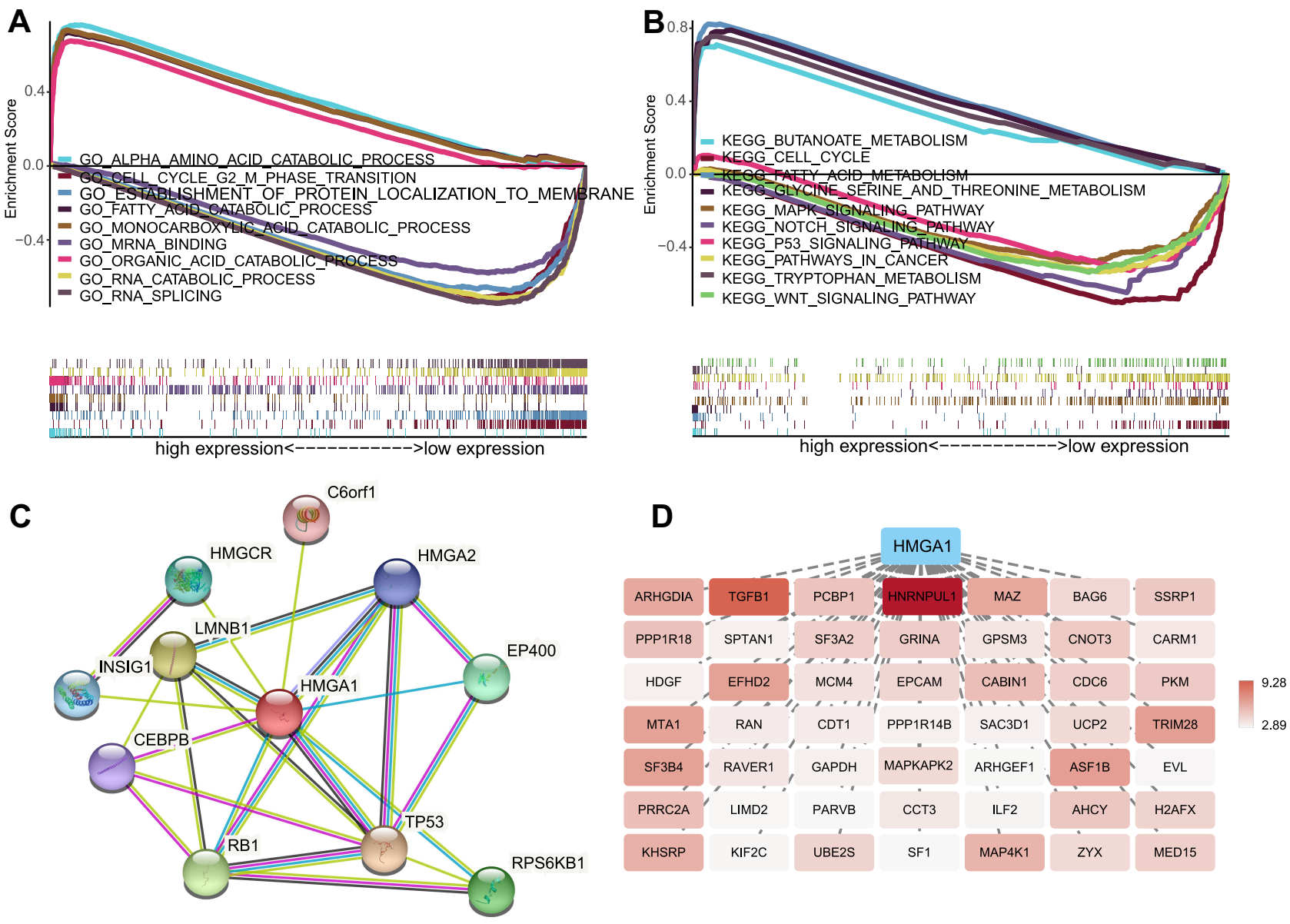

Figure 4 Significantly enriched GO annotations (A) and KEGG pathways (B) of HMGAI in HCC based on TCGA database. (C) PPI analysis of HMGA by STRING. (D) HMGAI co-expression network was inferred from Coexpedia and deep hue indicated a higher degree of association with HMGAI.

Abbreviations: GO, Gene Ontology; KEGG, Kyoto Encyclopedia of Genes and Genomes; PPI, Protein-protein interaction; HMGAI, high-mobility group AT-hook I; TCGA, The Cancer Genome Atlas; STRING, search tool for recurring instances of neighboring genes. 

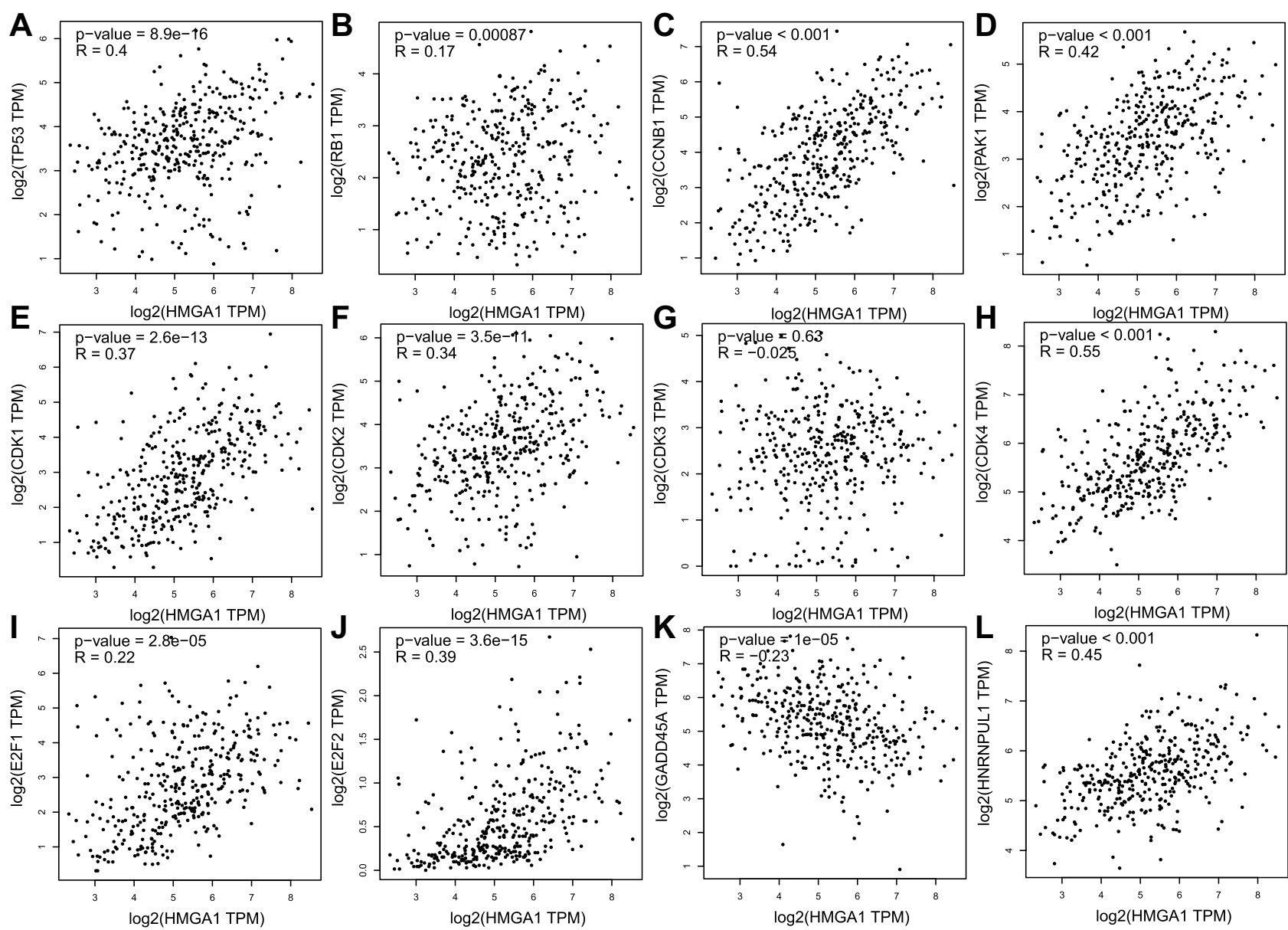

Figure 5 HMGAI mRNA expression was respectively significant positive correlation to the mRNA expressions of $T P 53(R=0.4, P<0.00 I), R B I(R=0.17, P<0.00 I)$, $C C N B I$ $(R=0.54, P<0.00 I)$, PAKI $(R=0.42, P<0.00 I), C D K I(R=0.37, P<0.00 I), C D K 2(R=0.34, P<0.00 I), C D K 4(R=0.55, P<0.00 I), E 2 F I(R=0.22, P<0.00 I), E 2 F 2(R=0.39$, $P<0.00 \mathrm{I})$, HNRNPULI $(R=0.45, P<0.00 \mathrm{I})$, while was significant negative correlation to GADD45A $(R=-0.23, P<0.00 \mathrm{I})(\mathbf{A}-\mathbf{L})$.

Abbreviations: HMGAI, high-mobility group AT-hook I; TP53, tumor protein p53; RBI, RB Transcriptional Corepressor I; CCNBI, Cyclin BI; PAKI, P2I (RACI) Activated Kinase I; CDKI, Cyclin Dependent Kinase I; CDK2, Cyclin Dependent Kinase 2; CDK4, Cyclin Dependent Kinase 4; E2FI, E2F Transcription Factor I; HNRNPULI, Heterogeneous Nuclear Ribonucleoprotein U Like I; GADD45A, Growth Arrest and DNA Damage Inducible Alpha.

remain primitive, poorly differentiated, and stem-like state in both cancer and normal development. ${ }^{41-43}$ Amounting studies proved that the suppression of oncogenic HMGA1 significantly inhibited some cancer cell proliferation and migration, providing a novel clue to probe into anti-cancer approaches. $^{21,29,32,44,45}$ Furthermore, HMGA1 is upregulated in a significant proportion of $\mathrm{HCC}$, and its overexpression is associated with a poor prognosis. A research in vitro models ulteriorly elucidated that HMGA1 overexpression may act a pivotal role in cell viability and migration and promote anchorage-independent growth to induce transformation in liver cancer cell lines. ${ }^{26,27}$ Consistently, our study demonstrated that HMGA1 expression was amplified in HCC and high-expression HMGA1 was closely associated with advanced pathological stage, unpromising T stage, and poor pathological differentiation. Accordingly, oncogenic properties and aggressive malignancy of HMGA1 were uncovered. Microtubule Interacting and Trafficking Domain containing 1 (MITD1) can serve as a novel predictor for human HCC prognosis, which had been demonstrated. By contrast, HMGA1 had a better prognostic evaluation effectiveness with a higher AUC. ${ }^{46}$

Recent studies had identified many frequently mutated genes in HCC genomic alterations, including TERT promoter, TP53 (tumor protein P53), and CTNNB1 (b-catenin) and so on, wherein TP53, a crucial cancer-driver mutation gene, accounted for about $30 \%$ of cases. ${ }^{1}$ As a tumor suppressor gene, TP53 activates its protein expression to regulate its downstream genes and subsequently inhibit malignant transformation by inducing cell cycle arrest, apoptosis, DNA repair, and senescence. ${ }^{47,48}$ Its mutation is significantly associated with the advanced 


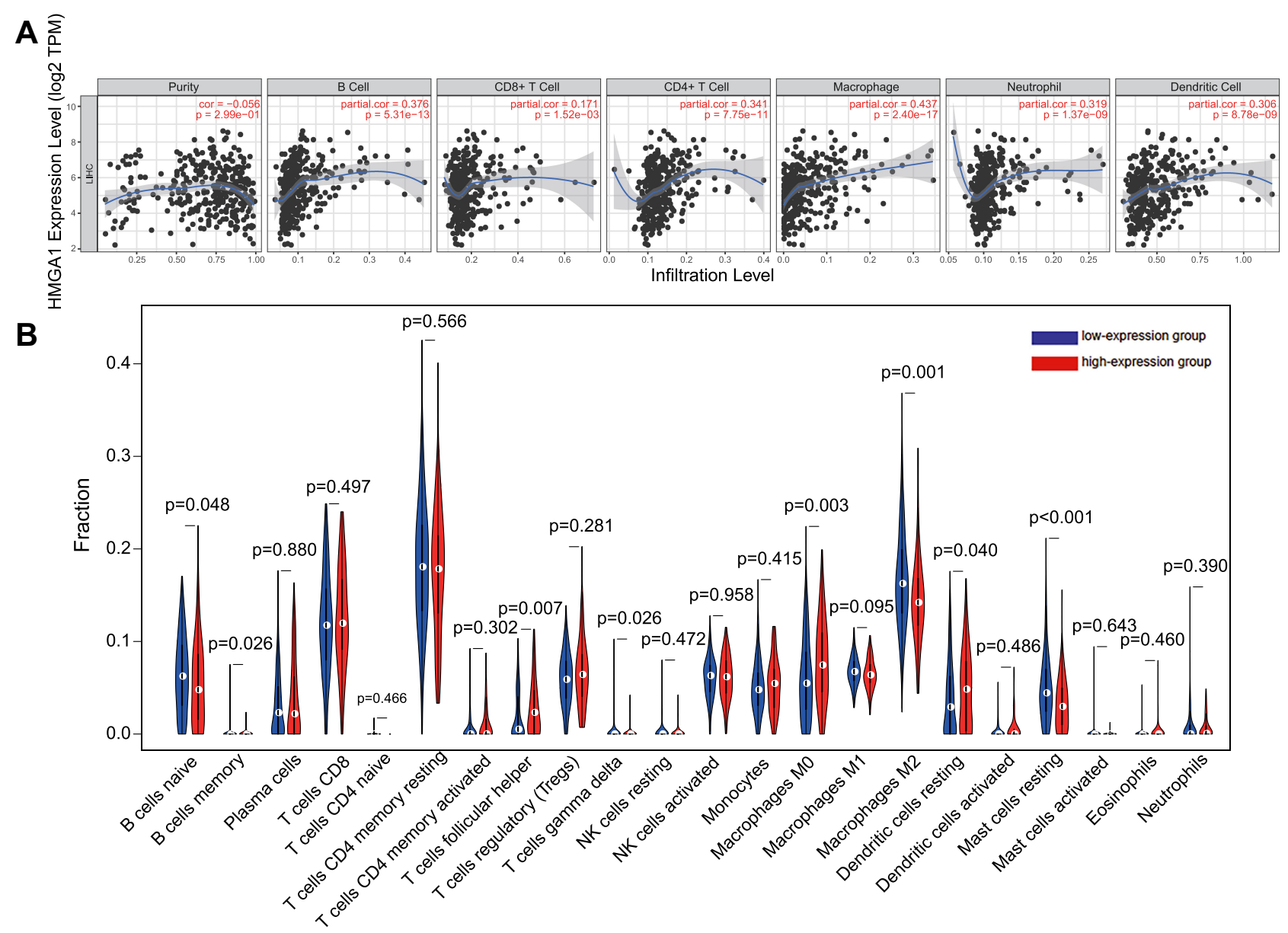

Figure 6 The relationship between HMGAI and immune invasion in hepatocellular carcinoma. (A) Relationship between the abundance of immune cells and HMGAI expression in HCC (TIMER). (B) Different proportions of sorts of immune cells in high- and low-HMGAI groups in HCC specimens (CIBERSORT).

Abbreviations: HMGAI, high-mobility group AT-hook I; HCC, hepatocellular carcinoma; TIMER, Tumor Immune Estimation Resource; CIBERSORT, Cell type Identification through the Estimating Relative Subsets of RNA Transcripts.

clinical stage, poor prognosis, and occurrence of liver cancer. ${ }^{49,50}$ HMGA1 regulated the 553 signaling pathway at the transcriptional level by regulating the balance between symmetric and asymmetric divisions in cancer stem cells and suppressing apoptosis, according to the new studies. ${ }^{31,42,51}$ Besides, hnRPUL1 was demonstrated to interact directly with $\mathrm{p} 53$, inhibit $\mathrm{p} 53$ transcriptional activity in response to UV radiation, and play a role in DNA damage response, especially in the process of alcohol-related cirrhosis. ${ }^{52,53}$ Additionally, substantial epidemiological and experimental evidence had revealed that HMGA1 may regulate carcinogenesis and development of cancer by activating the $\mathrm{Wnt} / \beta$-catenin pathway. ${ }^{20,22,30,54}$ Xian et al uncovered that such activating pattern conformed to a feed-forward amplification loop, whereby Wnt induces HMGA1, which in turn, enhances signaling. ${ }^{54}$ Moreover, $\beta$-catenin activation promotes immune evasion and resistance to anti-PD-1 therapy in
HCC. ${ }^{55}$ Our study demonstrated that high expression level HMGA1 phenotype closely linked with several cancer-related pathways (e.g. cell cycle, MAPK signaling pathway, NOTCH signaling pathway, VEGF signaling pathway, p53 signaling pathway, and WNT signaling pathway). Furthermore, HCC patients with TP53 dysregulation had higher HMGA1 expression than non-mutations, and HMGA1 was weakly - moderately correlated with TP53 downstream genes and hnRPUL1. Accordingly, it can be inferred that HMGA1 may exert an important effect on TP53 mutational carcinogenesis. These outcomes may explain why HMGA1 functions as an oncogene and its overexpression portends an aggressive malignancy of cancers.

As a crucial part of TME, the cellular composition of immune infiltration is tightly regulated by the various chemokines, which modulate tumor immunity and the biological phenotype of the tumors and further impact tumor progression, 


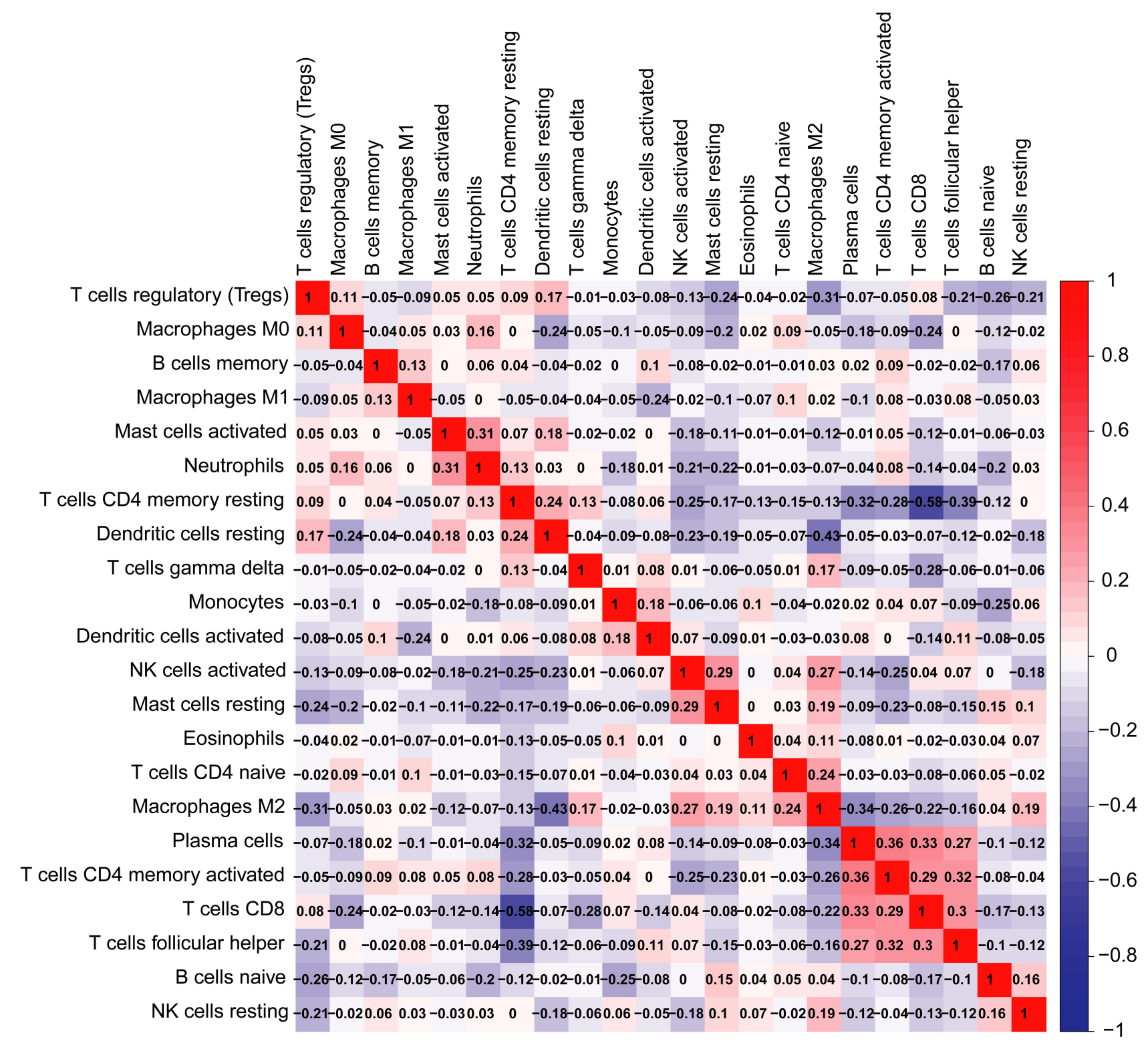

Figure 7 Resulting heat map visualizing the correlation matrix of diverse tumor-infiltrating immune cells subpopulations. Each tile indicates coefficients calculated by Pearson's correlation test.

therapy, and prognosis. ${ }^{1,12-14,36}$ These days, emerging insights into tumor biology suggested that successful therapeutics in the future depended on rescuing $\mathrm{T}$ cells from the homing and dysfunction state. Tumor-associated macrophages (TAM) function as a vital mediator of tissue homeostasis. Macrophages M1 activity inhibits cell proliferation and causes tissue damage, while M2 activity promotes cell proliferation and tissue repair. Adjuvant immunotherapy targeting macrophages was considered beneficial for HCC patients. ${ }^{56-59}$ Most cancers, including HCC, overexpress inhibitory ligands to evade the immune response by dampening $\mathrm{T}$ cell function, thus contributing to cancer progression. A study proved that by activating HMGA1-dependent signaling pathways including the PI3K/Akt and MEK/ERK pathways, PD-L1 maintained colorectal cancer stem cells and promoted the expansion of cancer. ${ }^{60}$ The results of this study demonstrated that HMGA1 immune infiltration in HCC. Remarkably, the amount of M0 macrophages displayed a significant rise in the high HMGA1 expression phenotype, while Macrophages M2 decreased. Accordingly, it was speculated that HMGA1 may exert dual influences on tumor-infiltrating immune system, namely recruitment and management of tumor-infiltrating cells and helping the immune evade by regulating TAM via activating of the relevant signal pathways. 


\section{Conclusions}

In conclusion, HCC with a high HMGA1 expression level may be the one with malignant nature and dismal prognosis. Moreover, excess levels of HMGA1 have a crucial role in the regulation of immune infiltrating cells and may be an accomplice to help the immune escape in HCC. However, precise approaches by which HMGA1 with multifaceted functions promotes hepatocarcinogenesis and links with liver cirrhosis or viral hepatitis need further investigation.

\section{Data Sharing Statement}

All raw online RNA sequencing dataset and clinical information of HCC patients, which were included in the current study, can be downloaded from the TCGA (https:// portal.gdc.cancer.gov/), ICGC (http://dcc.icgc.org), and GEO database (https://www.ncbi.nlm.nih.gov/geo/). The data that support the findings of this study are available from the corresponding author upon reasonable request.

\section{Ethics Statement}

The present study was approved by the Ethics Committee of The First Affiliated Hospital of Guangxi Medical University [Approval Number: 2021 (KY-E-032)] and all patients provided written informed consent. This trial was conducted in accordance with the ethical principles in the Declaration of Helsinki.

\section{Acknowledgments}

The authors thank the contributors of The Cancer Genome Atlas (https://cancergenome.nih.gov/), ICGC (http://dcc. icgc.org), and GEO database (https://www.ncbi.nlm.nih. gov/geo/) for sharing the HCC dataset on open access. In addition, we would like to acknowledge the helpful comments on this article received from our reviewers.

\section{Disclosure}

The authors declare that they have no competing interests.

\section{References}

1. Villanueva A, Longo DL. Hepatocellular carcinoma. $N$ Engl J Med. 2019;380(15):1450-1462. doi:10.1056/NEJMra1713263

2. Bray F, Ferlay J, Soerjomataram I, et al. Global cancer statistics 2018: GLOBOCAN estimates of incidence and mortality worldwide for 36 cancers in 185 countries. CA Cancer J Clin. 2018;68(6):394-424. doi:10.3322/caac. 21492

3. Zhou M, Wang H, Zeng X, et al. Mortality, morbidity, and risk factors in China and its provinces, 1990-2017: a systematic analysis for the Global Burden of Disease Study 2017. Lancet. 2019;394 (10204):1145-1158. doi:10.1016/S0140-6736(19)30427-1
4. Fujiwara N, Friedman SL, Goossens N, et al. Risk factors and prevention of hepatocellular carcinoma in the era of precision medicine. $J$ Hepatol. 2018;68(3):526-549. doi:10.1016/j. jhep.2017.09.016

5. Marengo A, Rosso C, Bugianesi E. Liver cancer: connections with obesity, fatty liver, and cirrhosis. Annu Rev Med. 2016;67:103-117. doi:10.1146/annurev-med-090514-013832

6. Dimitroulis D, Damaskos C, Valsami S, et al. From diagnosis to treatment of hepatocellular carcinoma: an epidemic problem for both developed and developing world. World $J$ Gastroenterol. 2017;23(29):5282-5294. doi:10.3748/wjg.v23.i29.5282

7. Baig B, Halim SA, Farrukh A, et al. Current status of nanomaterial-based treatment for hepatocellular carcinoma. Biomed Pharmacother. 2019;116:108852. doi:10.1016/j.biopha.2019.108852

8. Benassi E, Fan H, Sun Q, et al. Generation of particle assemblies mimicking enzymatic activity by processing of herbal food: the case of rhizoma polygonati and other natural ingredients in traditional Chinese medicine. Nanoscale Adv. 2021;3(8):2222-2235. doi:10.1039/D0NA00958J

9. Hamza AA, Heeba GH, Hamza S, et al. Standardized extract of ginger ameliorates liver cancer by reducing proliferation and inducing apoptosis through inhibition oxidative stress/ inflammation pathway. Biomed Pharmacother. 2021;134:111102. doi:10.1016/j. biopha.2020.111102

10. Xie Y, Mu C, Kazybay B, et al. Network pharmacology and experimental investigation of Rhizoma polygonati extract targeted kinase with herbzyme activity for potent drug delivery. Drug Deliv. 2021;28 (1):2187-2197. doi:10.1080/10717544.2021.1977422

11. Nazarbek G, Kutzhanova A, Nurtay L, et al. Nano-evolution and protein-based enzymatic evolution predicts novel types of natural product nanozymes of traditional Chinese medicine: cases of herbzymes of Taishan-Huangjing (Rhizoma polygonati) and Goji (Lycium chinense). Nanoscale Adv. 2021;3(23):6728-6738. doi:10.1039/ D1NA00475A

12. Sim HW, Knox J. Hepatocellular carcinoma in the era of immunotherapy. Curr Probl Cancer. 2018;42(1):40-48. doi:10.1016/j.currproblcancer.2017.10.007

13. Pinato DJ, Fessas P, Sapisochin G, et al. Perspectives on the neoadjuvant use of immunotherapy in hepatocellular carcinoma. Hepatology. 2020. doi: 10.1002/hep.31697

14. Wu X, Gu Z, Chen Y, et al. Application of PD-1 blockade in cancer immunotherapy. Comput Struct Biotechnol J. 2019;17:661-674. doi:10.1016/j.csbj.2019.03.006

15. Akinleye A, Rasool Z. Immune checkpoint inhibitors of PD-L1 as cancer therapeutics. J Hematol Oncol. 2019;12(1):92. doi:10.1186/ s13045-019-0779-5

16. Barcena-Varela M, Lujambio A. The endless sources of hepatocellular carcinoma heterogeneity. Cancers. 2021;13(11):2621. doi:10.3390/cancers 13112621

17. Stephen B, Hajjar J. Overview of basic immunology and clinical application. Adv Exp Med Biol. 2020;1244:1-36. doi:10.1007/9783-030-41008-7_1

18. Dong LQ, Peng L-H, Ma L-J, et al. Heterogeneous immunogenomic features and distinct escape mechanisms in multifocal hepatocellular carcinoma. $J$ Hepatol. 2020;72(5):896-908. doi:10.1016/j. jhep.2019.12.014

19. Gajewski TF, Schreiber H, Fu YX. Innate and adaptive immune cells in the tumor microenvironment. Nat Immunol. 2013;14 (10):1014-1022. doi:10.1038/ni.2703

20. Han X, Cao Y, Wang K, et al. HMGA1 facilitates tumor progression through regulating Wnt/ $\beta$-catenin pathway in endometrial cancer. Biomed Pharmacother. 2016;82:312-318. doi:10.1016/j. biopha.2016.05.004

21. Qi C, Cao J, Li M, et al. HMGA1 overexpression is associated with the malignant status and progression of breast cancer. Anat Rec. 2018;301(6):1061-1067. doi:10.1002/ar.23777 
22. Xing J, Cao G, Fu C. HMGA1 interacts with $\beta$-catenin to positively regulate $\mathrm{Wnt} / \beta$-catenin signaling in colorectal cancer cells. Pathol Oncol Res. 2014;20(4):847-851. doi:10.1007/s12253-014-9763-0

23. Sumter TF, Xian L, Huso T, et al. The high mobility group A1 (HMGA1) transcriptome in cancer and development. Curr Mol Med. 2016;16(4):353-393. doi:10.2174/15665240166661 60316152147

24. Nakanishi F, Ohkawa $\mathrm{K}$, Ishida $\mathrm{H}$, et al. Alteration in gene expression profile by full-length hepatitis B virus genome. Intervirology. 2005;48(2-3):77-83. doi:10.1159/000081732

25. Zhang Z, Yao Z, Zhao S, et al. Interaction between autophagy and senescence is required for dihydroartemisinin to alleviate liver fibrosis. Cell Death Dis. 2017;8(6):e2886. doi:10.1038/ cddis. 2017.255

26. Chang ZG, Yang L-Y, Wang W, et al. Determination of high mobility group A1 (HMGA1) expression in hepatocellular carcinoma: a potential prognostic marker. Dig Dis Sci. 2005;50(10):1764-1770. doi:10.1007/s10620-005-2934-9

27. Andreozzi M, Quintavalle C, Benz D, et al. HMGA1 expression in human hepatocellular carcinoma correlates with poor prognosis and promotes tumor growth and migration in in vitro models. Neoplasia. 2016;18(12):724-731. doi:10.1016/j.neo.2016.10.002

28. Chuma M, Saeki N, Yamamoto Y, et al. Expression profiling in hepatocellular carcinoma with intrahepatic metastasis: identification of high-mobility group $\mathrm{I}(\mathrm{Y})$ protein as a molecular marker of hepatocellular carcinoma metastasis. Keio J Med. 2004;53(2):90-97. doi: $10.2302 / \mathrm{kjm} .53 .90$

29. Di Cello F, Shin J, Harbom K, et al. Knockdown of HMGA1 inhibits human breast cancer cell growth and metastasis in immunodeficient mice. Biochem Biophys Res Commun. 2013;434(1):70-74. doi:10.1016/j.bbrc.2013.03.064

30. Resar L, Chia L, Xian L. Lessons from the crypt: HMGA1-amping up Wnt for stem cells and tumor progression. Cancer Res. 2018;78 (8):1890-1897. doi:10.1158/0008-5472.CAN-17-3045

31. Frasca F, Rustighi A, Malaguarnera R, et al. HMGA1 inhibits the function of p53 family members in thyroid cancer cells. Cancer Res. 2006;66(6):2980-2989. doi:10.1158/0008-5472.CAN-05-2637

32. Schuldenfrei A, Belton A, Kowalski J, et al. HMGA1 drives stem cell, inflammatory pathway, and cell cycle progression genes during lymphoid tumorigenesis. BMC Genomics. 2011;12:549. doi:10.1186/ 1471-2164-12-549

33. Pak K, Oh SO, Goh TS, et al. A user-friendly, web-based integrative tool (ESurv) for survival analysis: development and validation study. J Med Internet Res. 2020;22(5):e16084. doi:10.2196/16084

34. Szklarczyk D, Gable AL, Lyon D, et al. STRING v11: protein-protein association networks with increased coverage, supporting functional discovery in genome-wide experimental datasets. Nucleic Acids Res. 2019;47(D1):D607-D613. doi:10.1093/nar/gky1131

35. Shannon P, Markiel A, Ozier O, et al. Cytoscape: a software environment for integrated models of biomolecular interaction networks. Genome Res. 2003;13(11):2498-2504. doi:10.1101/gr.1239303

36. Li B, Severson E, Pignon J-C, et al. Comprehensive analyses of tumor immunity: implications for cancer immunotherapy. Genome Biol. 2016;17(1):174. doi:10.1186/s13059-016-1028-7

37. Li T, Fan J, Wang B, et al. TIMER: a web server for comprehensive analysis of tumor-infiltrating immune cells. Cancer Res. 2017;77(21): e108-e110. doi:10.1158/0008-5472.CAN-17-0307

38. Newman AM, Liu CL, Green MR, et al. Robust enumeration of cell subsets from tissue expression profiles. Nat Methods. 2015;12 (5):453-457. doi:10.1038/nmeth.3337

39. Wang X, Liao X, Yu T, et al. Analysis of clinical significance and prospective molecular mechanism of main elements of the JAK/ STAT pathway in hepatocellular carcinoma. Int J Oncol. 2019;55 (4):805-822. doi:10.3892/ijo.2019.4862

40. Pallante P, Sepe R, Puca F, et al. High mobility group a proteins as tumor markers. Front Med. 2015;2:15. doi:10.3389/fmed.2015.00015
41. Shah SN, Kerr C, Cope L, et al. HMGA1 reprograms somatic cells into pluripotent stem cells by inducing stem cell transcriptional networks. PLoS One. 2012;7(11):e48533. doi:10.1371/journal. pone. 0048533

42. Puca F, Colamaio M, Federico A, et al. HMGA1 silencing restores normal stem cell characteristics in colon cancer stem cells by increasing p53 levels. Oncotarget. 2014;5(10):3234-3245. doi:10.18632/ oncotarget. 1914

43. Parisi S, Piscitelli S, Passaro F, et al. HMga proteins in stemness and differentiation of embryonic and adult stem cells. Int $J$ Mol Sci. 2020;21(1).

44. Shah SN, Cope L, Poh W, et al. HMGA1: a master regulator of tumor progression in triple-negative breast cancer cells. PLoS One. 2013;8 (5):e63419.

45. Hassan F, Lossie SL, Kasik EP, et al. A mouse model study of toxicity and biodistribution of a replication defective adenovirus serotype 5 virus with its genome engineered to contain a decoy hyper binding site to sequester and suppress oncogenic HMGA1 as a new cancer treatment therapy. PLoS One. 2018;13(2):e0192882. doi:10.1371/journal.pone. 0192882

46. Shen H, Wang Z, Ren S, et al. Prognostic biomarker MITD1 and its correlation with immune infiltrates in hepatocellular carcinoma (HCC). Int Immunopharmacol. 2020;81:106222. doi:10.1016/j. intimp.2020.106222

47. Muller PA, Vousden KH. p53 mutations in cancer. Nat Cell Biol. 2013;15(1):2-8. doi:10.1038/ncb2641

48. Murali C, Mudgil P, Gan C-Y, et al. Camel whey protein hydrolysates induced G2/M cellcycle arrest in human colorectal carcinoma. Sci Rep. 2021;11(1):7062. doi:10.1038/s41598-021-86391-z

49. Xue R, Li R, Guo H, et al. Variable intra-tumor genomic heterogeneity of multiple lesions in patients with hepatocellular carcinoma. Gastroenterology. 2016;150(4):998-1008. doi:10.1053/j. gastro.2015.12.033

50. Takai A, Dang HT, Wang XW. Identification of drivers from cancer genome diversity in hepatocellular carcinoma. Int J Mol Sci. 2014;15 (6):11142-11160. doi:10.3390/ijms 150611142

51. Pierantoni GM, Rinaldo C, Esposito F, et al. High mobility group A1 (HMGA1) proteins interact with p53 and inhibit its apoptotic activity. Cell Death Differ. 2006;13(9):1554-1563. doi:10.1038/sj. cdd. 4401839

52. Barral PM, Rusch A, Turnell AS, et al. The interaction of the hnRNP family member E1B-AP5 with p53. FEBS Lett. 2005;579 (13):2752-2758. doi:10.1016/j.febslet.2005.03.095

53. Innes $\mathrm{H}$, Buch $\mathrm{S}$, Hutchinson $\mathrm{S}$, et al. Genome-wide association study for alcohol-related cirrhosis identifies risk loci in MARC1 and HNRNPUL1. Gastroenterology. 2020;159(4):1276-1289.e7. doi:10.1053/j.gastro.2020.06.014

54. Xian L, Georgess D, Huso T, et al. HMGA1 amplifies Wnt signalling and expands the intestinal stem cell compartment and Paneth cell niche. Nat Commun. 2017;8:15008. doi:10.1038/ ncomms 15008

55. Ruiz de Galarreta M, Bresnahan E, Molina-Sánchez P, et al. $\beta$-catenin activation promotes immune escape and resistance to Anti-PD-1 therapy in hepatocellular carcinoma. Cancer Discov. 2019;9 (8):1124-1141. doi:10.1158/2159-8290.CD-19-0074

56. DeNardo DG, Ruffell B. Macrophages as regulators of tumour immunity and immunotherapy. Nat Rev Immunol. 2019;19(6):369-382. doi:10.1038/s41577-019-0127-6

57. Xia Y, Rao L, Yao H, et al. Engineering macrophages for cancer immunotherapy and drug delivery. Adv Mater. 2020;32(40): e2002054. doi:10.1002/adma.202002054

58. Degroote H, Van Dierendonck A, Geerts A, et al. Preclinical and clinical therapeutic strategies affecting tumor-associated macrophages in hepatocellular carcinoma. $J$ Immunol Res. 2018;2018:7819520. doi:10.1155/2018/7819520 
59. Mantovani A, Marchesi F, Malesci A, et al. Tumour-associated macrophages as treatment targets in oncology. Nat Rev Clin Oncol. 2017;14(7):399-416. doi:10.1038/nrclinonc.2016.217
60. Wei F, Zhang T, Deng S-C, et al. PD-L1 promotes colorectal cancer stem cell expansion by activating HMGA1-dependent signaling pathways. Cancer Lett. 2019;450:1-13. doi:10.1016/j.canlet.2019.02.022

\section{Publish your work in this journal}

The International Journal of General Medicine is an international, peer-reviewed open-access journal that focuses on general and internal medicine, pathogenesis, epidemiology, diagnosis, monitoring and treatment protocols. The journal is characterized by the rapid reporting of reviews, original research and clinical studies across all disease areas. The manuscript management system is completely online and includes a very quick and fair peer-review system, which is all easy to use. Visit http://www.dovepress.com/ testimonials.php to read real quotes from published authors.

Submit your manuscript here: https://www.dovepress.com/international-journal-of-general-medicine-journal 